\title{
Cloacal adenocarcinoma and mycobacteriosis in a White's Tree Frog - case description
}

\author{
ALEKSANDRA LEDWOŃ, IZABELLA DOLKA, BEATA DOLKA, \\ ANDRZEJ ŻYCZYŃSKI*, WOJCIECH BIELECKI
}

\begin{abstract}
Department of Pathology and Veterinary Diagnostics, Faculty of Veterinary Medicine, Warsaw University of Life Sciences, Nowoursynowska 159c St., 02-776 Warsaw, Poland

*Department of Genetics and Animal Breeding, Faculty of Animal Sciences, Warsaw University of Life Sciences, Ciszewskiego 8 St., 02-787 Warsaw, Poland
\end{abstract}

\section{Ledwoń A., Dolka I., Dolka B., Życzyński A., Bielecki W. \\ Cloacal adenocarcinoma and mycobacteriosis in a White's Tree Frog - case description \\ Summary}

Mycobacterium fortuitum infection and adenocarcinoma were associated with cloacal prolapse in a White's tree frog (Litoria caerulea). The disease was characterized by the presence of nodules in a cloacal mucous membrane. Bacteriological examination of the cloacal tissue revealed the presence of Enterococcus sp., $E$. coli and numerous acid-fast bacteria (AFB). The presence of AFB was confirmed by a Ziehl-Neelsen stain of the cloacal tissue and culture on the Löwenstein-Jensen medium. Post-mortem examination showed no changes typical of mycobacteriosis or neoplasia in other organs.

Keywords: mycobacteriosis, adenocarcinoma, cloacal prolapse, White's tree frog

\section{Case description}

A ten-year-old female White's tree frog (Litoria caerulea) with cloacal prolapse was submitted for clinical examination. Oedema and congestion of the cloacal mucous membrane was observed, with poorly visible nodules of approximately $0.4 \mathrm{~cm}$ in diameter. Moreover, lack of corneal transparency was observed (Fig. 1). The frog was kept together with three other individuals in a terrarium generously watered twice a day and equipped with one

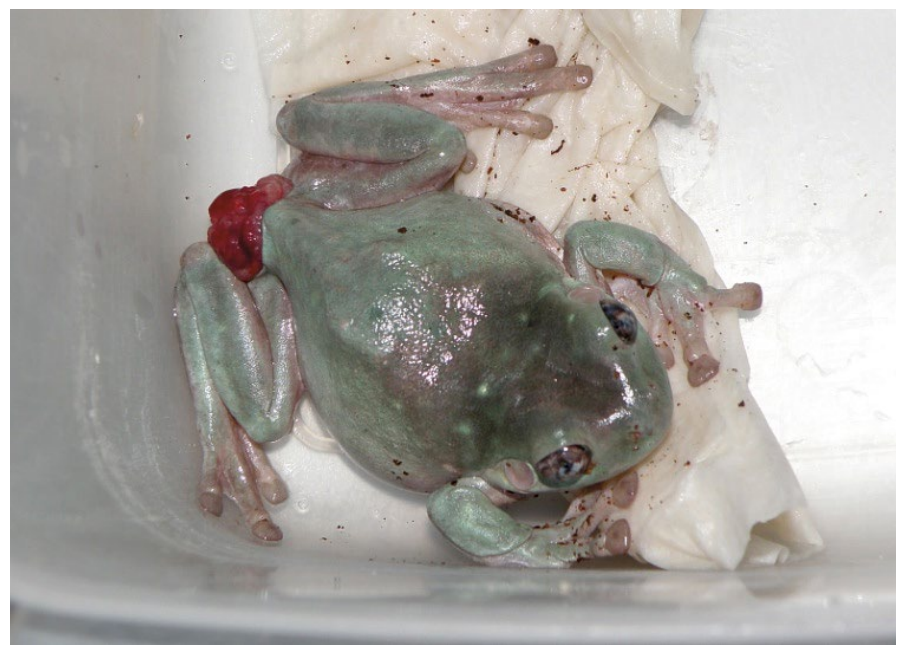

Fig. 1. Clinical presentation of cloacal prolaps and corneal lipidosis in White's Tree Frog heated side wall and a bulb. The ambient temperature was $25-30^{\circ} \mathrm{C}$. Daylight duration was $12 \mathrm{~h}$. The frogs were fed using tweezers twice a week, mainly with mouse neonates, and less frequently with bees and cockroaches. Lack of corneal transparency was found in the other two females, but it was not found in the significantly thinner male.

The cloaca was washed and disinfected with $0.3 \%$ gentamicin solution, after which it was replaced. For the following 4 days marbofloxacin (Marbocyl, Vetoquinol, France) was administered IM SID at $2 \mathrm{mg} / \mathrm{kg}$ and meloxicam (Metacam, Boehringer Ingelheim Vetmedica $\mathrm{GmbH}$, Germany) was administered IM SID at $0.2 \mathrm{mg} / \mathrm{kg}$. After a few days the cloaca prolapsed again, was replaced, and then prolapsed for a third time. In light of the further development of the nodular lesions on the prolapsed cloacal mucosa, euthanasia was decided. The frog was anesthesized with ketamine $100 \mathrm{mg} /$ $\mathrm{kg}$ bw (Ketamina 10\%, Biowet Pulawy, Poland) with xylazine $10 \mathrm{mg} / \mathrm{kg}$ bw (Sedazin, Biowet Pulawy, Poland) in an IM injection (6), and subsequently administered $20 \mathrm{mg}$ of pentobarbital sodium (Morbital, Biowet Pulawy, Poland) into the median abdominal vein. Immediately after death the frog was necropsied.

During necropsy, the gut was taken for bacteriological examination, and sections of the liver, kidneys, ovary, skeletal muscle, heart, cloaca and eye were taken for histopathological examination (hematoxylin-eosin staining). The nodular lesions covered only the prolapsed area of the cloaca. No lesions in other organs were observed in the 


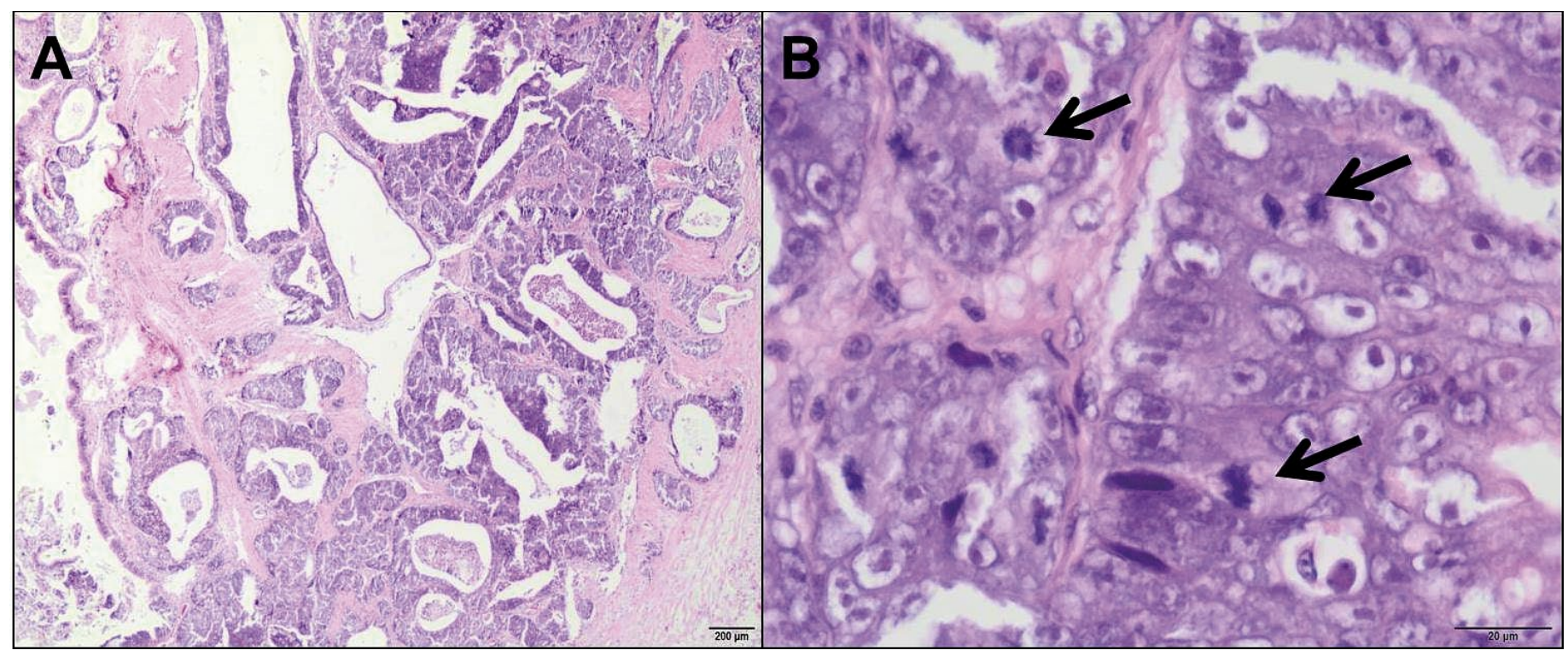

Fig. 2. A. Cloacal adenocarcinoma characterized by varied architectural patterns including tubular or solid diffuse sheets of cells. HE, $20 \times$. B. Adenocarcinoma was comprised of diffuse sheets of poorly differentiated epithelial cells, with many mitotic figures (arrows). HE, $400 \times$

necropsy. Fragments of the gut and cloaca were ground using mortar and pestle, resuspended in physiological saline and decontaminated using the Sputofluor preparation (Merck, Germany). The material was inoculated on the Löwenstein-Jensen PACT medium (Becton Dickinson, Sparks, USA) and incubated at $24^{\circ} \mathrm{C}$ and $37^{\circ} \mathrm{C}$. In a cloaca specimen stained using the Ziehl-Neelsen method (Merck KGaA, Darmstadt, Germany) numerous acid-fast bacilli were observed. The growth of the bacilli on the medium was obtained after approximately 10 days.

The intestinal swab was subjected to bacteriological examination in order to detect aerobic bacteria. The culture led to the isolation of Enterococcus sp., susceptible to amoxicillin, amoxicillin with clavulanic acid and florfenicol, and resistant to gentamicin, enrofloxacin, neomycin, colistin, tetracycline, cefatoxime, lincomycin, erythromycin and penicillin. Moreover, E. coli moderately susceptible to florfenicol and resistant to amoxicillin, amoxicillin with clavulanic acid, gentamicin, enrofloxacin, neomycin, colis-

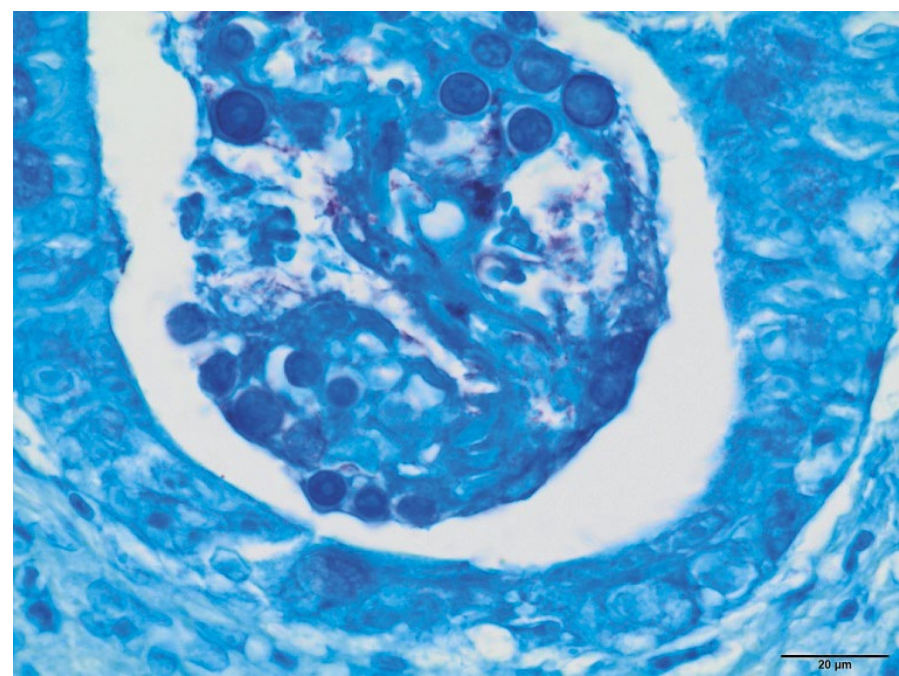

Fig. 3. Cellular debris and acid-fast organisms in tubular lumen of cloacal adenocarcinoma Ziehl-Neelsen, $400 \times$ tin, tetracycline, cefatoxime and sulfamethoxazole with trimethoprim, was isolated.

The histopathological examination revealed fragmentation and atrophy of cardiomyocytes and focal granules of melanin in the cardiac muscle; a large amount of melanin pigment in the hepatic parenchyma (a normal component of many amphibians) and sparse focal mononuclear infiltration. The cloacal mucosa was lined with simple columnar epithelium. Below, within fibrous connective tissue stroma tubulovesicular and solid structures composed of multiple epithelial cells with features of malignancy (atypia) were found. Moreover, a high mitotic index was observed (Fig. 2). Focal presence of cellular detritus in the lumen of the tubules was detected, and single bacilli stained with the Ziehl-Neelsen method could be seen in only one tubule (Fig. 3). Moreover, moderate interstitial inflammation with oedema of the cloacal mucosa was found. In the kidneys, vacuolization of the tubular epithelial cells was observed, with brown pigment distributed multifocally in the epithelial cells. In the corneas, cholesterol clefts surrounded by mild mononuclear infiltration and stromal melanin deposition were detected (Fig. 4).

The cultured acid-fast bacilli were subjected to DNA extraction using 5\% Chelex (Biorad, USA). Subsequently, PCR was conducted to identify Mycobacterium sp. (13), and the obtained product was sequenced to identify $\mathrm{Myco}-$ bacterium fortuitum subsp. fortuitum.

Cloacal swabs were taken from the other individuals for inoculation on the Löwenstein-Jensen PACT medium. No growth of acid-fast bacilli was obtained from these cultures.

\section{Discussion}

To date, no case of a tumour occurring simultaneously with a Mycobacterium fortuitum subsp. fortuitum infection has been reported in anurans, but similar diagnoses have been made in humans (2). The diagnosis of tumours in humans preceded the diagnosis of a very quick colonisation by rapidly growing AFB or, 


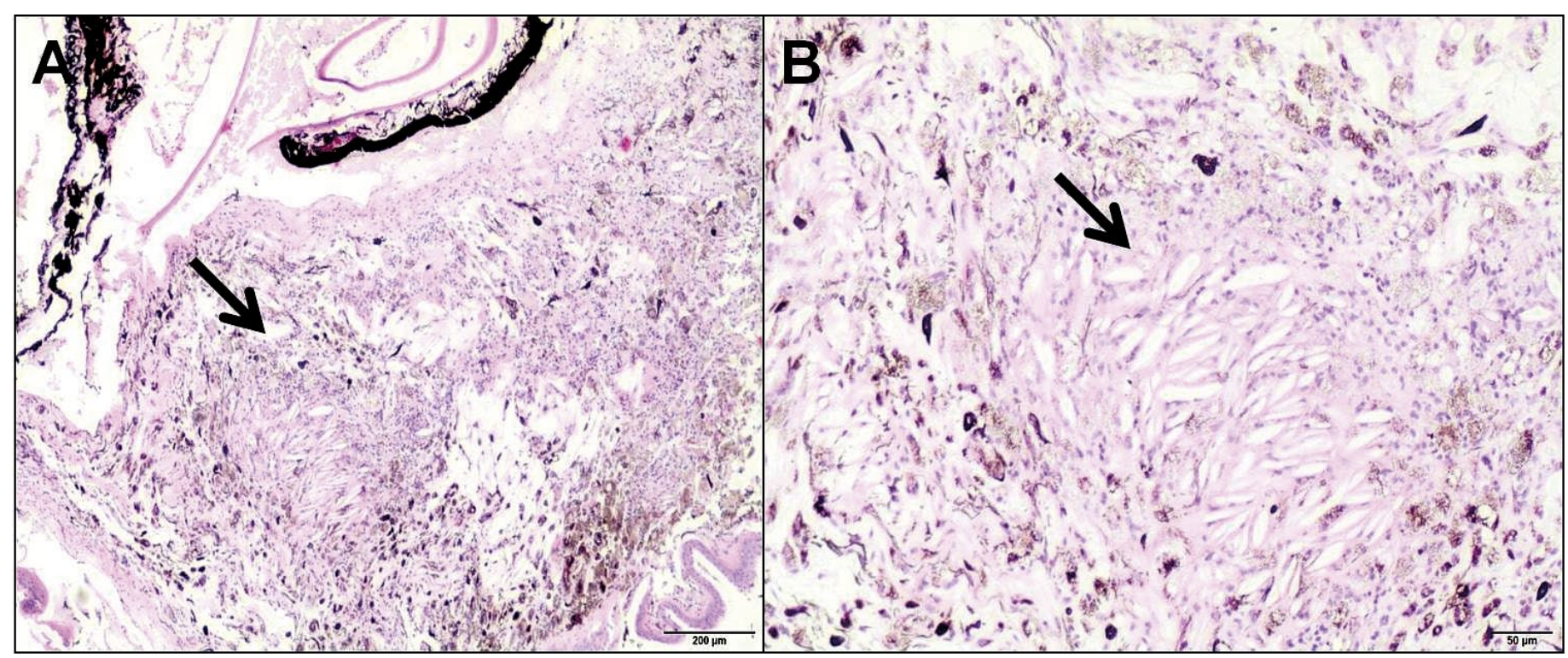

Fig. 4. A. Corneal lipidosis in the anterior stroma (arrow). HE, $40 \times$. B. Cholesterol cleft formation (arrow), melanin (brown) granules and mononuclear infiltration were noted. HE, $100 \times$

less frequently, mycobacteriosis. Factors predisposing to infection were antineoplastic chemotherapy and previous pulmonary involvement with malignant disease, including localized catheter-associated infections $(1,8)$. The opposite process was observed in tuberculosis, in which lung cancer often develops in the scars caused by Mycobacterium tuberculosis (11). Amphibian mycobacterioses are relatively common and are mainly caused by Mycobacterium ulcerans. Amphibians can also constitute a reservoir of these bacteria, which poses danger to human health (12). Interestingly, studies conducted in laboratory mice demonstrated an inhibiting effect of the M. ulcerans toxin on the development of mammary gland adenocarcinoma (3). Corneal lipidosis in anurans kept in captivity is diagnosed relatively frequently $(5,7,9$, 14). In its typical course, hypercholesterolaemia is a systemic disease associated with the formation of cholesterol deposits accompanied by inflammation in many organs $(9,14)$. The disease is caused by feeding amphibians with mouse neonates and house crickets that have a different lipid profile compared to natural food $(5,14)$. In the presented case, changes in the corneas occurred only in females that demonstrated a better body condition compared to the male. According to Holmberg et al. (4), lipid keratopathy occurs commonly in female anurans and may be associated with oogenesis; however, no egg deposition was observed either in this case or in the cases of diseased females described by other authors $(5,10)$. In view of the fact that the frogs were fed using tweezers, no signs of emaciation associated with blindness, i.e. due to the inability to find food, were observed.

\section{References}

1. Apiwattankul N., Flynn P. M., Hayden R. T., Adderson E. E.: Infections Caused by Rapidly Growing Mycobacteria spp in Children and Adolescents With Cancer. J. Pediatric Infect. Dis. Soc. 2015, 4, 104-113.
2. Feld R., Bodey G. P., Gröschel D.: Mycobacteriosis in patients with malignant disease. Arch. Intern. Med. 1976, 136, 67-70.

3. Heggers J. P., Robson M. C., Yetter J. F., Kreig R. E. Jr, Jennings P. B.: Tumoricidal effects of Mycobacterium ulcerans toxin on murine adenocarcinoma (C3HBA). J. Surg. Oncol. 1979, 11, 161-169.

4. Holmberg B. D.: Ophtalmology in Exotic Pets, [in:] Maggs D. J., Miller P., Douglas R. O., Slatter H.: Slatter's Fundamentals of Veterinary Ophthalmology. Saunders, St Louis Missouri 2008, p. 427-441.

5. Keller C. B., Shilton C. M.: The amphibian eye. Vet. Clin. North. Am. Exot. Anim. Pract. 2002, 5, 261-274.

6.Longley L. A.: Amphibian anaesthesia, [in:] Anaesthesia of Exotic Pets. Saunders Elsevier. London, UK 2008, p. 45-260.

7. Millichamp N. J., Dziezyc J., Anderson R. E., Maude M. B.: Lipid keratopathy in frogs: histopathology and biochemistry. Invest. Ophthalmol. Vis. Sci. 1990, 31,542 .

8. Rolston K. V., Jones P. G., Fainstein V., Bodey G. P.: Pulmonary disease caused by rapidly growing mycobacteria in patients with cancer. Chest. 1985, 87, 503-506.

9. Russell W. C., Edwards D. L., Stair E. L., Hubner D. C.: Corneal lipidosis, disseminated xanthomatosis and hypercholesterolemia in Cuban tree frog. J. Zoo Wildl. Med. 1990, 21, 99-104.

10. Shilton C. M.: Corneal lipid deposition in anurans [thesis]. University of Guelph, Canada 2000.

11. Tamura A.: Tuberculosis and lung cancer. Kekkaku 2016, 91, 17-25.

12. Willson S. J., Kaufman M. G., Merritt R. W., Williamson H. R., Malakauskas D. M., Benbow M. E.: Fish and amphibians as potential reservoirs of Mycobacterium ulcerans, the causative agent of Buruli ulcer disease. Infect. Ecol. Epidemiol. 2013, 3.doi: 10.3402/iee.v3i0.19946.

13. Wilton S., Cousins D.: Detection and identification of multiple mycobacterial pathogens by DNA amplification in a single tube. PCR Methods Appl. 1992, 1, 269-273.

14. Wright K.: Overview of amphibian medicine, [in:] Mader D. R.: Reptile Medicine and Surgery. Sauders Elsevier USA 2006, 941-968.

Corresponding author: Aleksandra Ledwoń, DVM PhD, Nowoursynowska 159c St., 02-776 Warsaw, Poland; e-mail: aleksandra_ledwon@sggw.pl 